\title{
Origin of the Parry arc
}

Article

Published Version

Westbrook, C. (2011) Origin of the Parry arc. Quarterly Journal of the Royal Meteorological Society, 137 B. pp. 538-543. ISSN 1477-870X doi: https://doi.org/10.1002/qj.761 Available at https://centaur.reading.ac.uk/20663/

It is advisable to refer to the publisher's version if you intend to cite from the work. See Guidance on citing.

To link to this article DOI: http://dx.doi.org/10.1002/qj.761

Publisher: Royal Meteorological Society

All outputs in CentAUR are protected by Intellectual Property Rights law, including copyright law. Copyright and IPR is retained by the creators or other copyright holders. Terms and conditions for use of this material are defined in the End User Agreement.

\section{www.reading.ac.uk/centaur}

\section{CentAUR}

Central Archive at the University of Reading

Reading's research outputs online 


\title{
RMetS
}

\section{Notes and correspondence Origin of the Parry arc}

\author{
C. D. Westbrook ${ }^{\star}$ \\ Department of Meteorology, University of Reading, Reading, UK \\ ${ }^{\star}$ Correspondence to: Dr Chris Westbrook, Department of Meteorology, University of Reading, Earley Gate, PO Box 243, \\ Reading, RG66BB, UK. E-mail: c.d.westbrook@reading.ac.uk
}

\begin{abstract}
Laboratory experiments to determine the preferred orientation of free-falling hexagonal prisms were performed at Reynolds numbers appropriate to falling ice crystals in the atmosphere. Hexagonal plates orient with their $c$ axis vertical for aspect ratios $<0.9$, whilst hexagonal columns fall with their $c$ axis horizontal. A secondary alignment is also observed: regular hexagonal columns fall preferentially with two prism facets aligned vertically and not horizontally - the latter scenario was previously assumed to be responsible for the rare Parry arc. However, if the column is made scalene in its cross-section, it can orient such that a pair of prism facets is horizontal. This finding indicates that the development of scalene crystals may be key to the production of certain ice-crystal optical phenomena. Copyright (C) 2011 Royal Meteorological Society
\end{abstract}

Key Words: ice crystal; optical effects; particle orientation

Received 10 August 2010; Revised 1 December 2010; Accepted 2 December 2010; Published online in Wiley Online Library 23 February 2011

Citation: Westbrook CD. 2011. Origin of the Parry arc. Q. J. R. Meteorol. Soc. 137: 538-543. DOI:10.1002/qj.761

\section{Introduction}

It is well known that ice crystals in free fall often adopt a certain preferred orientation. This has implications for radiative transfer (Takano and Liou, 1989), lidar observations (Westbrook et al., 2010), and the formation of halos and other optical effects (Tricker, 1970). In particular, the rare Parry arc has long been attributed to hexagonal columns falling with a pair of prism faces lying in the horizontal plane (Tricker, 1970; Ulanowski, 2005), yet the conditions for this special orientation remain unknown (Tape, 1994). Based on the analysis of scanning polarization lidar measurements, Sassen and Takano (2000) have suggested that this 'Parry orientation' may be a special property of the fluid flow around thick hexagonal plate crystals: however, they point out that laboratory experiments are needed to test this hypothesis. The purpose of this note is to communicate the results of such experiments and to give some insight into the crystal shapes likely responsible for this optical phenomenon. Observations of hexagonal prisms falling in a tank of dense fluid were used to determine their preferred orientations and some details of the flow patterns at Reynolds numbers appropriate to natural ice crystals. The prisms fell stably and steadily, and the fluid flow around these analogues is therefore simply a scaled-up version of the air flow around an ice crystal falling stably and steadily in air at the same Reynolds number (Tritton, 1988).

\section{Experimental set-up}

The hexagonal prisms are high-impact polystyrene rods produced commercially for hobby/modelling purposes. Various thicknesses (nominally 3, 1.5, 1 and $0.8 \mathrm{~mm}$ span between opposite prism faces) were used in order to investigate a wide range of Reynolds numbers. The prisms were cut to length using a Stanley knife, and their ends filed flat. Aspect ratios investigated ranged from 0.25 (plate) to 25 (long, thin column). Exact dimensions were measured using a pair of digital Vernier calipers. The spans across all three pairs of opposing prism faces were measured, and were found to be equal to within the accuracy of the instrument $(0.01 \mathrm{~mm})$, 


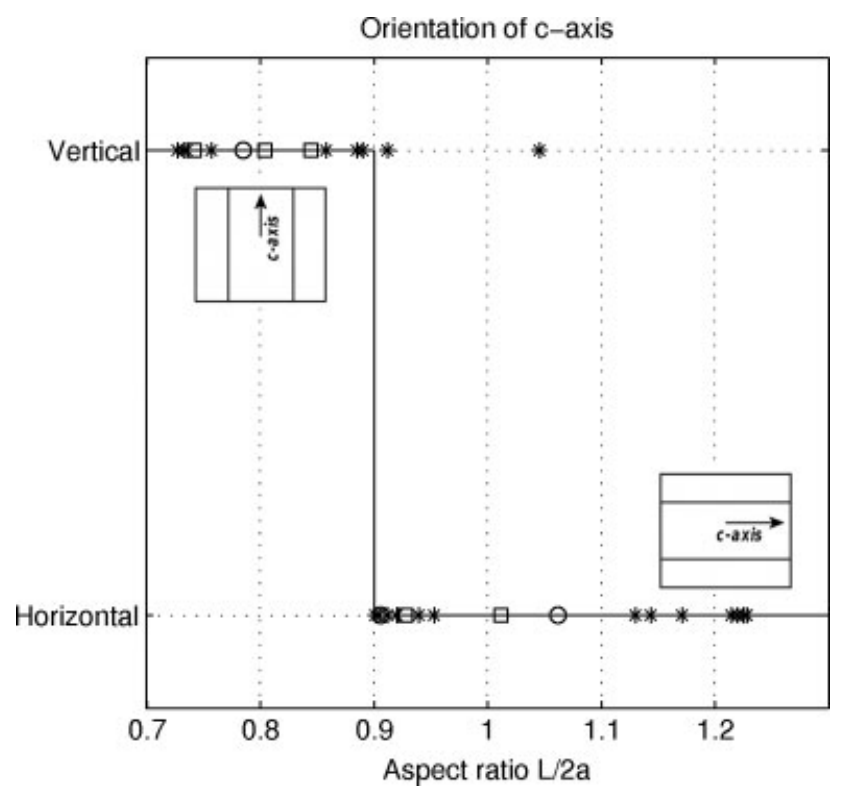

Figure 1. Primary orientation of free-falling hexagonal prisms as a function of aspect ratio. Stars are for particles falling at $R e \approx 100$; squares show additional data at $R e=35$ and circles show data for $R e=8$.

\section{(i) Parry orientation}
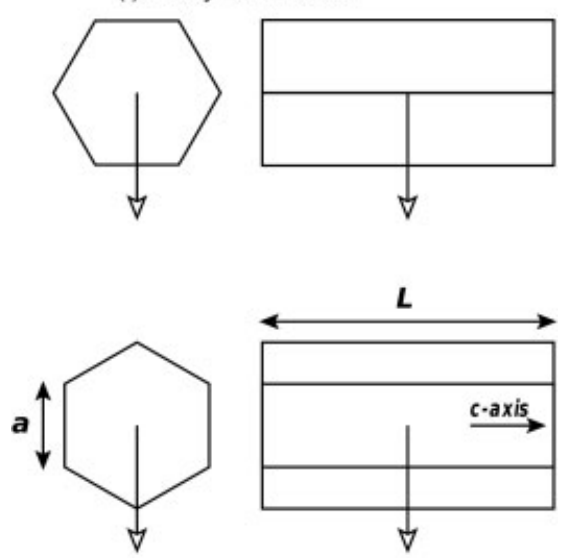

(ii) alternative orientation

Figure 2. Diagram showing possible orientations of falling hexagonal columns: (i) Parry-oriented where two prism facets lie in a horizontal plane; (ii) alternative orientation where two prism facets lie in a vertical plane. End- and side-on views are shown for each orientation. Arrows with open arrowheads indicate the direction of fall.

confirming that the prism cross-section was a regular hexagon.

The model crystals were dropped into the centre of a large cylindrical tank $(29 \mathrm{~cm}$ in diameter, $60 \mathrm{~cm}$ high $)$, filled with water or glycerine/water mixtures. This setup allowed plenty of time for the models to rotate to their preferred orientation, and the large diameter of the tank allows the neglect of wall effects. The time taken for each model to fall a $25 \mathrm{~cm}$ length of the tank was measured using a stopwatch and this information was used to determine the terminal velocity and therefore the Reynolds number of the models. A thermometer was used to determine the temperature of the fluid, and from this the density and viscosity were estimated (Weast, 1988).

The Reynolds number characterizes the relative importance of inertial and viscous forces, and is defined as

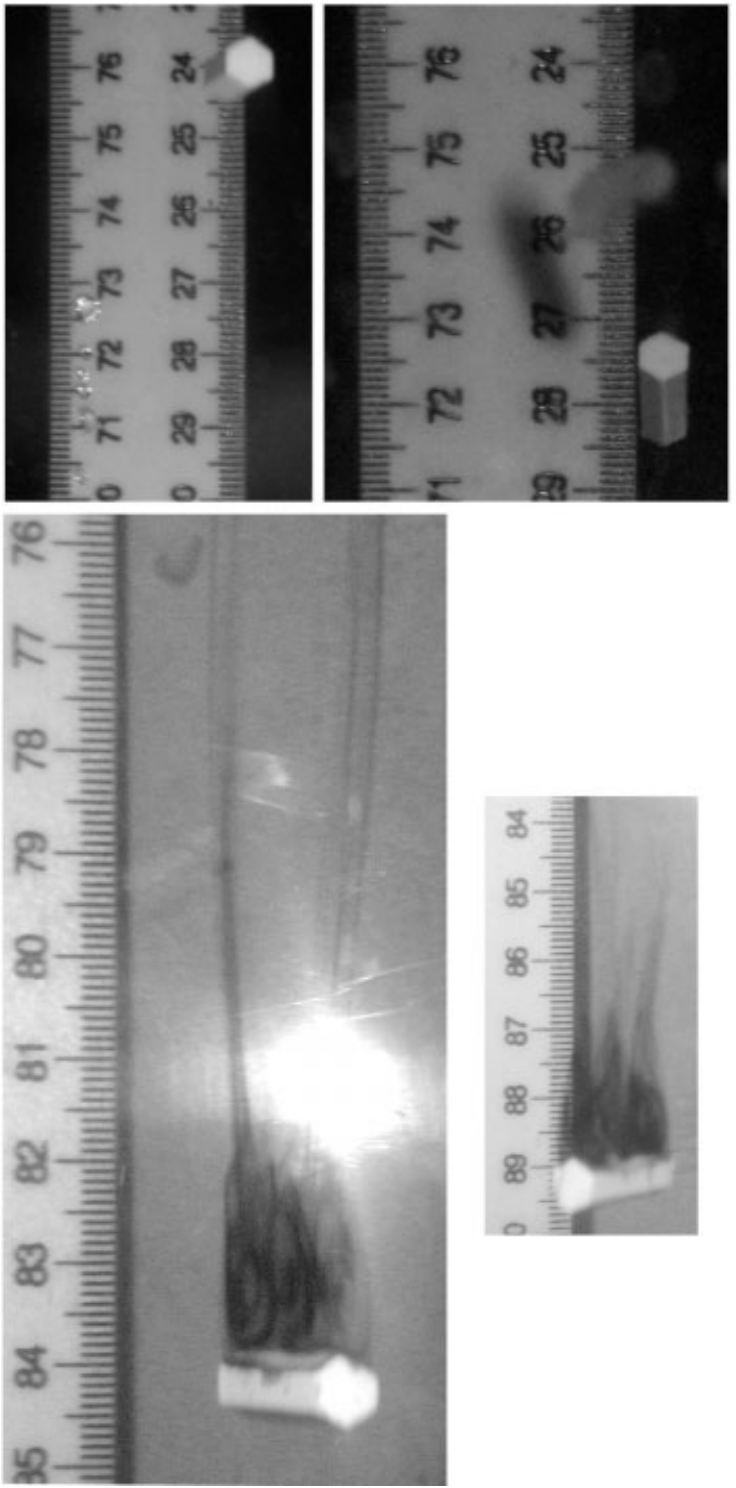

Figure 3. Photographs of regular hexagonal prisms falling with two prism facets vertical. The ruler in the background is aligned vertically behind the tank. Note the effect of viewing angle: in all cases the prism's $c$ axis was horizontal. The lower panel shows experiments in which the prisms were coated with dye in order to visualise the fluid flow at the rear of the particle; $R e=100$.

$R e=v d / \nu_{k}$ for a particle falling at terminal velocity $v$ in a fluid with kinematic viscosity $v_{k}$, where $d$ is the maximum span across the hexagonal cross-section of the prism. Ice crystals in the atmosphere may span a wide range of $R e$ from $<0.1$ when newly formed to $\sim 100$ when they are a few millimetres in length. When $R e$ is small, inertial forces are weak: reorientation occurs slowly and in the atmosphere may be disrupted by rotational Brownian motion and turbulence. At Reynolds numbers larger than $\sim 1$, inertial forces are significant, and it is these inertial forces that act to rotate the particles into their preferred orientation. The experiments here therefore focus on the latter regime, with Reynolds number varying over the range $R e \approx 2-100$.

\section{Results using regular hexagonal prisms}

A total of $\approx 150$ runs were performed with models of various thickness, aspect ratio and fluid density/viscosity. 


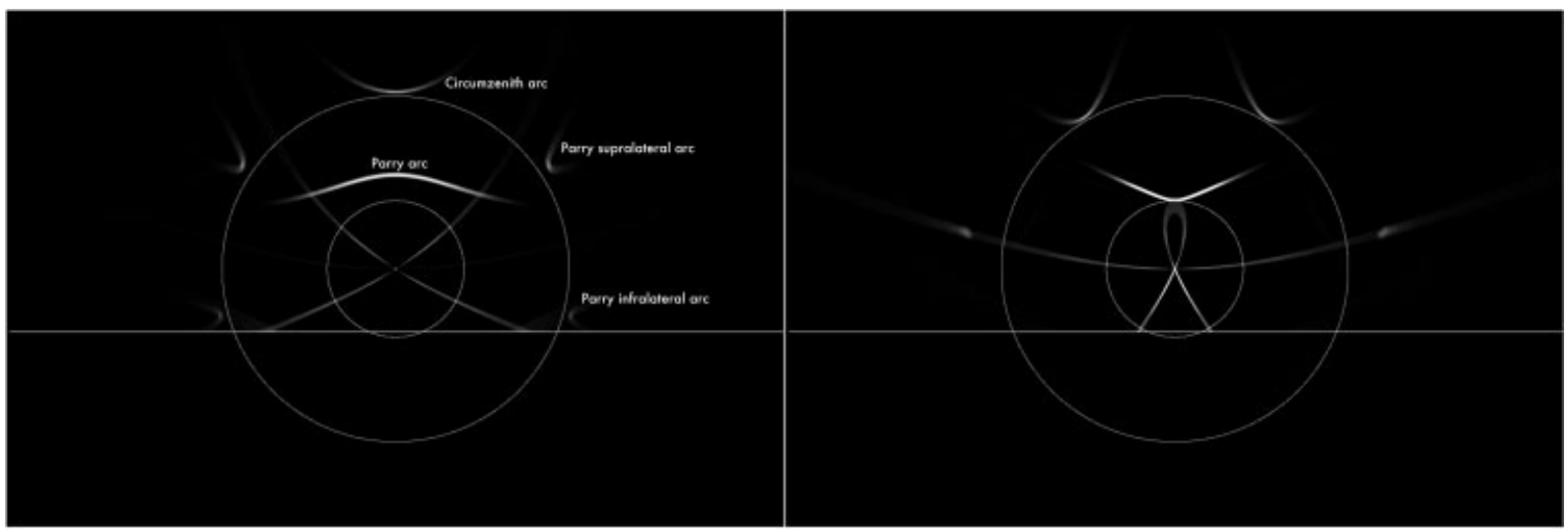

Figure 4. Ray-tracing simulations of the scattering pattern produced by regular hexagonal columns with two facets horizontal (left panels) and two facets vertical (right panels) as viewed by a $35 \mathrm{~mm}$ camera with an $8 \mathrm{~mm}$ lens centred on the sun. Results are shown for a solar elevation angle of $20^{\circ}$. Concentric circles around the sun mark the position of 22 and $46^{\circ}$ haloes for reference; the horizontal line indicates the horizon. Note that the intensity scale has been exaggerated to bring out the weaker features such as the ribbon-shaped arc intersecting the sun in the right-hand panel: in practice these features would be extremely faint.

\section{Scalene cross-sections}
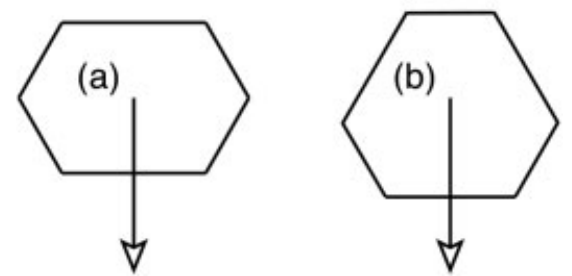

Figure 5. Diagram showing the two kinds of scalene column investigated here, viewed end-on: (a) flattened hexagon with four short sides and two long sides; (b) hexagon with three short sides and three long sides. All of the external angles are $60^{\circ}$; arrows indicate the direction of fall.

In what follows the prism is characterized by the span across the basal face $d=2 a$ and its length $L$ parallel to the $c$ axis (see Figure 2); the aspect ratio is defined here as $L / d$.

\subsection{Primary orientation}

All of the models fell stably. Hexagonal plates with $L / d \ll 1$ always reoriented themselves such that their $c$ axis was vertical, irrespective of their initial orientation, while hexagonal columns with aspect ratios $\gg 1$ always reoriented themselves with their $c$ axis horizontal. These results are in keeping with the behaviour of circular cylinders (Kajikawa, 1971). To determine the critical aspect ratio at which the preferred orientation changes, a series of experiments were carried out on prisms with aspect ratios close to 1. Figure 1 shows the orientation of prisms with aspect ratios in the range $0.7-1.3$. For this experiment the thickest $(3 \mathrm{~mm})$ rods were used, and the Reynolds number was $\approx 100$. Apart from a single outlier, there appears to be a well-defined transition at $L / d=0.9$ : below this value hexagonal prisms fall with their $c$ axis vertical, while above this value horizontal orientation of the $c$ axis is preferred. The single outlier data point is likely the result of an unobserved air bubble attached to a basal face of the prism.

In principle this critical aspect ratio could be a function of Reynolds number. To test this, additional data were collected at $R e=35$ and $R e=8$ and these are overlaid on Figure 1. No $R e$ dependence was found: the new data were found to be consistent with a critical value of $L / d=0.9$.

\subsection{Secondary orientation}

Unlike circular cylinders, it may be preferential for a hexagonal column with $L / d>0.9$ to align itself not only such that its $c$ axis lies horizontally, but also so that its hexagonal cross-section is one of the two orientations illustrated in Figure 2: these orientations allow a symmetrical flow pattern around the crystal. Orientation (i) is referred to as Parry-oriented, and is widely believed to be responsible for the Parry arc (Tricker, 1970).

Intriguingly, it was observed that hexagonal columns fell in orientation (ii) rather than the Parry orientation, with a pair of prism faces aligned vertically in all of the experiments performed here (total of $\approx 130$ runs where $L / d>0.9$ ); aspect ratios spanned the range $0.9<L / d<25$ and Reynolds numbers $2<R e<100$. This orientation could be confirmed clearly by eye for the larger models, and example photographs are shown in Figure 3. Note the effect of viewing angle in these photographs: in all cases the particle $c$ axis was horizontal. For the smaller models, every other prism face was marked with a light coating of graphite so that orientations (i) and (ii) could easily be distinguished by looking down on the falling particle from the top of the tank. Experiments in which the models were coated with water-soluble dye (black food colouring) allowed some details of the flow pattern behind the column to be inferred. At low Reynolds numbers (small models) it was difficult to observe any clear structure beyond a narrow sheet of dye trailing directly behind the top edge of the falling column. At larger Reynolds numbers, two pairs of stable, almond-shaped eddies inclined at an angle to the direction of fall became clearly visible, each pair tapering to a trailing wake: example photographs are shown in Figure 3.

\subsection{Implication for the Parry arc and other optical effects}

The results above show that regular hexagonal columns preferentially orient with two prism faces aligned vertically and not horizontally. This leads to the production of a different set of optical displays (Greenler, 1980). Example ray-tracing simulations are shown for a $20^{\circ}$ solar elevation in Figure 4, alongside results for the traditional Parry orientation. To the author's knowledge, the displays 

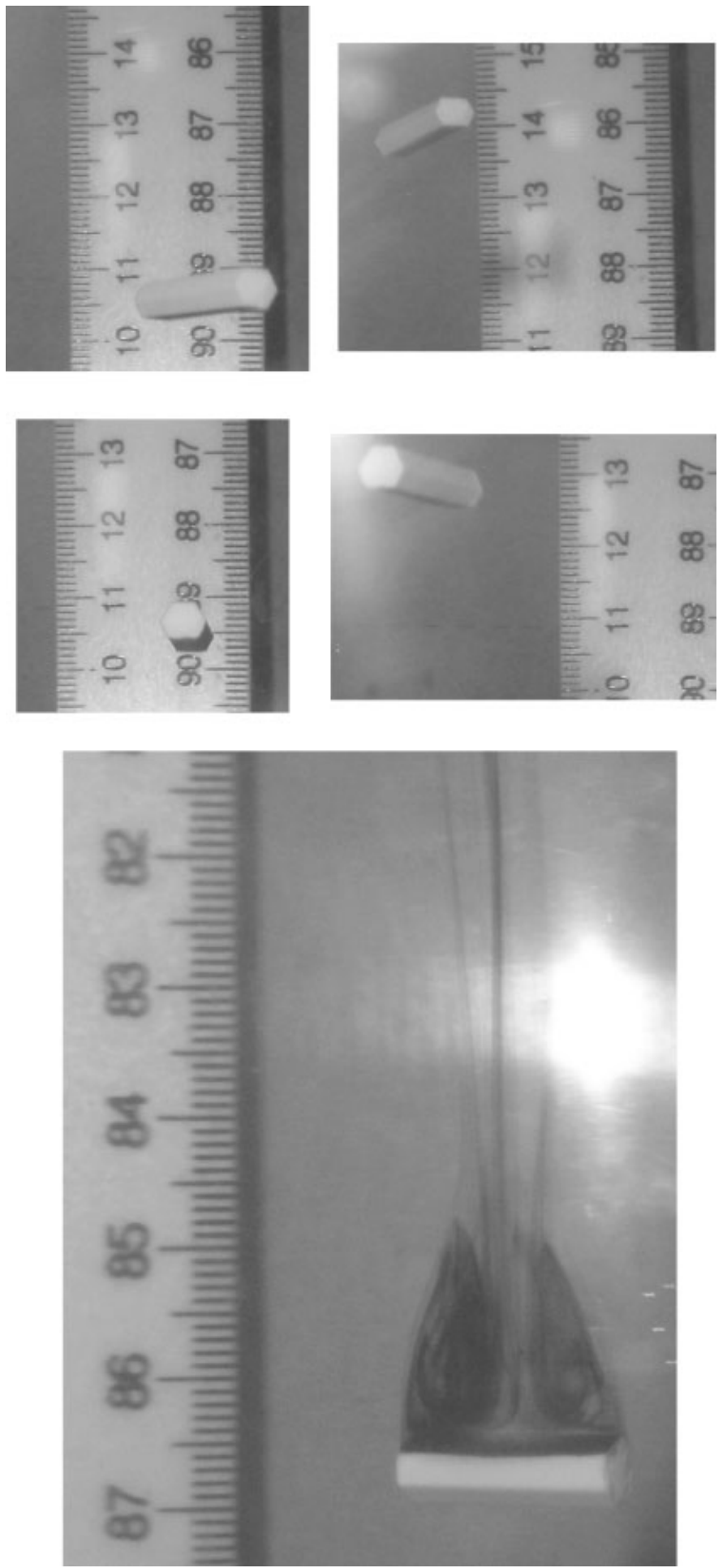

Figure 6. Photographs of free-falling scalene hexagonal prisms: the ruler is oriented vertically, and all particles shown are Parry-oriented. Note the effect of viewing angle - in all cases the $c$ axis was horizontal. The lower panel shows an experiment in which a scalene prism of type (b) was coated with dye in order to visualise the fluid flow at the rear of the particle.

associated with orientation (ii) have not been observed in the atmosphere, suggesting that the torque associated with the secondary orientation observed above is very weak and easily disrupted by turbulence or crystal imperfections. It is also possible that the primary $\mathrm{v}$-shaped arc might be mistaken for a weak upper tangent arc in some circumstances, or indeed be masked by the presence of a brighter upper tangent arc. In any case, the puzzle remains: what is responsible for the Parry arc?

\section{Scalene crystals}

Tricker (1970) suggested that Parry orientation might be favoured by a distorted, flattened prism cross-section. While his suggested geometry was extreme, hexagonal ice crystals can often take a scalene form (Yamashita, 1973), in which the sides of the hexagonal cross-section are no longer all equal but the external angles between the sides are fixed at $60^{\circ}$. In fact, Bailey and Hallett (2004) argue that 
scalene particles are more likely to occur than regular crosssections. To investigate the effect of this, some of the larger models were modified to produce two scalene types: (a) a flattened or 'tabular' form in which a pair of opposite prism faces were filed down to produce a cross-section with two long sides and four short sides; (b) a model where every other prism face was filed down to give a cross-section with three long sides and three short sides. These types are shown schematically in Figure 5, and the proportions shown are approximately those used in the experiment.

For both scalene types (a) and (b) there was a clear reorientation of the models such that two prism faces are aligned horizontally (Parry-oriented), as illustrated in Figure 5. Photographs of the particles in free fall are presented in Figure 6 and confirm this behaviour. Again, dye was applied to visualise the flow at the rear of the falling particle, and as for the regular hexagonal prisms four almond-shaped standing eddies were observed: an example photograph for a type (b) prism is shown in Figure 6.

Since all of the angles between the crystal faces are the same for scalene crystals as for regular hexagonal columns, the light rays will follow the same path to form the Parry arc as had been previously hypothesised for regular columns, albeit the arc will be slightly weaker in strength because of the reduced size of the lower inclined prism faces. It therefore seems highly likely that it is in fact these scalene crystals that are responsible for the formation of the Parry arc in nature.

Some more asymmetrical models were also experimented with. Type (a) prisms that were flattened unequally relative to their centre of gravity tended to oscillate around the Parry orientation. Cross-sections with six unequal sides were typically unstable at large $R e$; at low $R e$ these models did not rotate to position (i) or (ii) but instead assumed an intermediate secondary orientation. More detailed investigations into how the orientation is controlled by the distribution of weight are planned for future work.

\section{Conclusions}

The results presented here strongly suggest that regular hexagonal columns are not responsible for the Parry arc and other associated optical phenomena. No evidence was found to support Sassen and Takano's (2000) hypothesis that thick plates with $0.75<L / d<0.93$ are responsible for the Parry arc: hexagonal plates with $L / d<0.9$ orient with their $c$ axis aligned vertically, and regular prisms with $L / d>0.9$ do not assume the Parry orientation in any case. It appears that it is in fact scalene hexagonal columns that yield the flow pattern necessary to sustain the Parry orientation, and that only relatively modest changes from the regular hexagonal cross-section are needed to produce this effect: these changes are certainly within the range of crystal shapes observed in the atmosphere.

The above conclusion is also supported by crystals sampled during Parry-arc events. Tape (1994) sampled crystals at the ground at the South Pole during several Parry-arc displays, and these (in particular displays 2-4, 3-1, 3-4) show scalene columns in abundance, particularly the flattened type. Similarly, Sassen and Takano (2000) presented images of some of the crystals sampled in the cirrus cloud that produced their Parry arc: a number of these crystals appear to be short scalene columns of type (b). It would be valuable to perform new ray-tracing calculations to see whether Sassen and Takano (2000)'s depolarisation ratio scans could be explained by Parry-oriented scalene columns.

It is interesting to consider the sensitivity of these secondary orientations to evaporation. An experiment was carried out in which the corners of a prism $(L / d=3$, $R e=35$ ) were rounded off, since evaporation often proceeds preferentially from the corners (Nelson, 1998). The particle was observed to maintain its preferred secondary orientation despite this modification. However, if evaporation proceeds to completely erase the faceted structure of the crystal, the arcs in Figure 4 will of course no longer be produced.

In the experimental set-up used here it was very easy to observe preferred orientations over a wide range of $R e$; however in the atmosphere the low density of air relative to ice means that the critical Reynolds number for vortex-shedding and crystal oscillation is shifted to lower values, whilst for small Reynolds numbers secondary orientational torques are weak relative to turbulence and rotational Brownian motion. It seems likely that the combined requirements of $R e$ lying within a certain optimal range; the need for specific scalene crystal shapes and that those crystals be transparent with well-formed facets conspire to make the Parry arc the rare event that it is.

\section{Acknowledgements}

I acknowledge $\mathrm{Mr}$ Mike Stroud, $\mathrm{Mr}$ Ian Read and the other laboratory staff at the Department of Meteorology for their kind help and patience setting up this experiment. I am grateful for the valuable comments received from three anonymous reviewers. Figure 4 was produced using the HaloPoint 2.0 software package, and I thank Jukka Ruoskanen for making it freely available online: http://www.kolumbus.fi/jukka.ruoskanen/HaloPoint 2.html

\section{References}

Bailey M, Hallett J 2004. Growth rates and habits of ice crystals between $-20^{\circ}$ and $-70^{\circ}$ C. J. Atmos. Sci. 61: 514-544.

Greenler R. 1980. Rainbows, halos and glories. Cambridge University Press: Cambridge, UK.

Kajikawa M. 1971. A model experimental study of the falling velocity of ice crystals. J. Meteorol. Soc. Jpn 49: 367-375.

Nelson J. 1998. Sublimation of ice crystals. J. Atmos. Sci. 55: 910-919.

Sassen K, Takano Y. 2000. Parry arc: a polarization lidar, ray-tracing, and aircraft case study. Appl. Opt. 39: 6738-6745.

Takano Y, Liou KN. 1989. Transfer of polarized infrared radiation in optically anisotropic media: application to horizontally oriented ice crystals. J. Atmos. Sci. 46: 20-36.

Tape W. 1994. Atmospheric Halos, Antarctic research series. American Geophysical Union: Washington DC, USA.

Tricker RAR. 1970. Introduction to meteorological optics. Mills and Boon: London.

Tritton DJ. 1988. Physical fluid dynamics. Oxford University Press: Oxford. 
Ulanowski Z. 2005. Ice analog halos. Appl. Optics 44: 5754-5758.

Weast RC (ed). 1988. Handbook of Chemistry and Physics, 69th Edition. CRC Press: Boca Raton, FL, USA.

Westbrook CD, Illingworth AJ, O'Connor EJ, Hogan RJ. 2010. Doppler lidar measurements of oriented planar ice crystals falling from supercooled and glaciated cloud layers. Q. J. R. Meteorol. Soc. 136: 260-276.

Yamashita A. 1973. On the trigonal growth of ice crystals. J. Meteorol. Soc. Jap. 61: 307-317. 